Document downloaded from:

http://hdl.handle.net/10251/68284

This paper must be cited as:

Mellado Arteche, M.; Skrzypczyk, K. (2013). Information fusion in multi-agent system based on reliability criterion. En Vision Based Systemsfor UAV Applications. Springer. 207-217. doi:10.1007/978-3-319-00369-6_13.

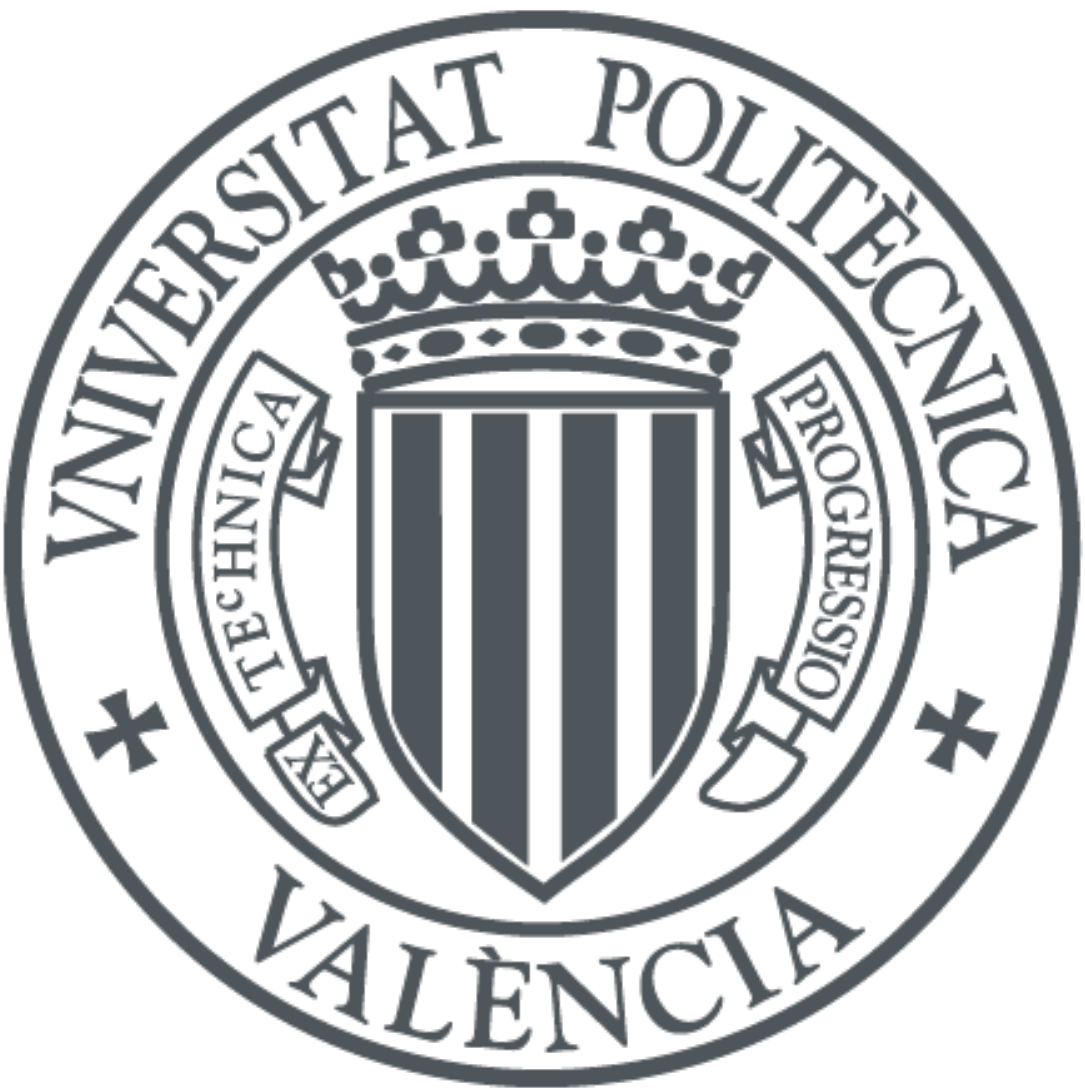

The final publication is available at

http://link.springer.com/chapter/10.1007/978-3-319-00369-6_13

Copyright Springer

Additional Information

The final publication is available at Springer via http://dx.doi.org/10.1007/978-3-319-003696_13 


\title{
Information Fusion in Multi-agent System Based on Reliability Criterion
}

\author{
Martin Mellado and Krzysztof Skrzypczyk
}

\author{
Martin Mellado \\ Instituto de Automatica e Informatica Industrial, Universidad Politecnica de Valencia \\ e-mail: krzysztof.skrzypczyk@polsl.pl, martin@ai2.upv.es
}

Krzysztof Skrzypczyk

Silesian University of Technology, Institute of Computer Science,

Akademicka 16, 44-101 Gliwice, Poland

\begin{abstract}
The paper addresses the problem of information fusion in Multi-Agent System. Since the knowledge of the process state is distributed between agents, the efficiency of the task performance depends on a proper information fusion technique applied to the agents. In this paper we study the case in which each agent has its own sensing device and is able to collect information with some certainty. Since the same information can be detected by multiple agents, the global certainty about the given fact derives from the fusion of information exchanged by interconnecting agents. The key issue in the method proposed, is an assumption that each agent is able to asses its own reliability during the task performance. The method is illustrated by the pick-up-and-collection task example. The effectiveness of the method proposed is evaluated using relevant simulation experiments.
\end{abstract}

\section{Introduction}

In Multi-Agent Systems (MAS), a primary task the system is intended to perform is distributed between a number of entities (agents). The beginnings of this branch of science were inspired by Artificial Intelligence in the 1950's. It was recognized that there are a number of tasks that MAS can perform more efficiently than centralized, single unit systems. Thanks to one of the features of MAS - modularity, even the domain of complex problems which are sometimes unpredictable can be solved by a number of simple entities, specialized in solving a particular part of the primary problem [12]. The benefits that these systems can offer are obvious 
and compelling. On the other hand, there are a lot of challenges that must be met in order to design effective and robust systems that are able to solve problems or execute tasks. These challenges were discussed in [12] and it is enough to point out the problems like coordination [2], [4],[5], [13], [14] task division [3], [7], [10],[17], cooperation [1], [7], [8], [15] etc. The potential advantages of MAS were quickly noticed by researchers who deal with problems related to Robotics. They have discovered that some specific tasks whose execution requires building and operating complex, powerful robotic units, can be performed more effectively by simpler, highly specialized robots. There are a number of problems that have become benchmarks and test-beds for the quality assessment and analysis of Multi-Robot Systems (MRS) functioning. This paper addresses one of the most important issues related to MAS which is fusion of information provided by multiple agents. The knowledge of the process state is distributed between agents, the efficiency of the task performance depends on a proper information fusion technique applied to the agents. In this paper we study the case in which each agent has its own sensing device and is able to collect information with some certainty. Since the same information can be detected by multiple agents, the global certainty about the given fact derives from the fusion of information exchanged by interconnecting agents. The key issue in the method proposed, is an assumption that each agent is able to assess its own reliability during the task performance. The method is illustrated by the pick-up-and-collection task example. However an application of the fusion technique discussed in this paper is not narrowed down only to the problem defined. It can be used in any system that is able to assess its own performance quality. A good example is the system of detecting and recognition objects by a number of cameras observing the same scene. Each of camera is usually mounted in an opto-electronic gimbal [16]. The effectiveness of the method proposed is evaluated using relevant simulation experiments.

\section{System Description}

The subject of this paper is the information fusion method in MAS. In order to present our approach we use the framework of the system dedicated for performing pick-up-and-collection task which consists of collecting a number of objects scattered inside a workspace of limited area. Figure 1 illustrates the principal idea of organizing the system. As was mentioned, there is an unknown number of objects to be collected, scattered over an area being the workspace of the system. The system consists of a specified number of robotic-units also called agents. Each agent can be defined by the following features [12]:

- limited sensing abilities,

- possibility of communication with limited number of other agents,

- a set of actions that an agent is able to take,

- reasoning about those actions based on its own knowledge. 
Since agents can communicate with each other they can exchange information about objects that are detected by their sensing devices and this way increase their knowledge of the number and location of objects sensed by other team mates. The subject of the paper is the method of agents sensing fusion which results in improvement of the process global knowledge.

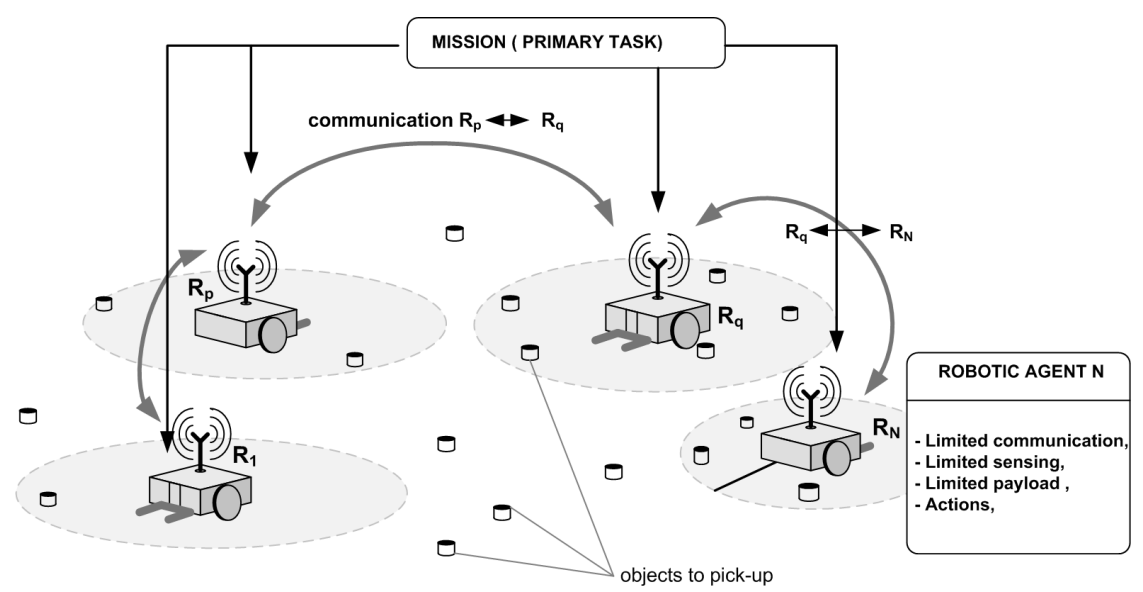

Fig. 1. Diagram that illustrated organization of the MAS being the subject of the discussion

\subsection{Workspace}

The workspace is a constrained, unstructured, flat area where a number of objects the robots are intended to collect are scattered. The objects are defined by a set of indices:

$$
O=\{i\}, \quad i=1,2, \ldots, M
$$

where $M$ is the total number of objects located inside the workspace. It has to be stressed that this number is not known to any agent. The location of each object is defined by a pair of coordinates $o_{i}=\left(x_{0, i}, y_{0, i}\right) i \in O$. No data of these positions are provided to particular agents.

\subsection{Agents Description}

The system consists of $N$ robotic agents that are equipped with a sensory system that allows them to detect objects $O$ and determine the location of detected objects. Let us define the team of the robotic agents as a set:

$$
R=\{i\}, \quad i=1,2, \ldots, N
$$


a pose $r_{i}$ of each agent is determined by a triple $\left(x_{r, i}, y_{r, i}, \Theta_{r, i}\right)$, where the elements denote coordinates of the center and heading of the agent defined in the workspace coordinate frame.

\subsection{Sensing Abilities}

Each robotic agent is provided with perception devices that allow it to detect objects in its vicinity. Therefore, let us define a set of objects detected by the ith agent as a set of indices:

$$
O_{i}=\{j\}, \quad i \in R, \quad j \in O \wedge O_{i} \subseteq O
$$

The perception of the agent is limited by the range of its sensors. The range of the sensors is denoted by $s_{r, i}$, which means that only those objects can be detected by the agents satisfying the following:

$$
k \in O_{i} \Leftrightarrow d_{i, k}^{o}=\sqrt{\left(x_{r, i}-x_{o, k}\right)^{2}+\left(y_{r, i}-y_{o, k}\right)^{2} \leq s_{r, i}}
$$

Each agent is able to assess its detection quality. It is defined by certainty factor which varies in range from 0 to 1 . The design of detection model and certainty evaluation is not the subject of this paper. For the purpose of the study we assume that detection certainty of the agent is influenced by two factors. The first one is related to its perception abilities which (it this study) decreases with the distance $d_{i, k}^{o}$ between the ith agent and $k t h$ object. The second factor influencing the certainty is a random process related to the sensing devices inaccuracy, noise etc. The influence level of particular factors is weighted by coefficients $w_{d}$ and $w_{n}$ the sum of which is equal to one. Using those coefficients we can simulate the sensing reliability of the given agent. In the study presented the detection certainty of the $k t h$ object by the ith agent is modeled as:

$$
\hat{p}_{i, k}=f_{i, k}^{d}+w_{n}\left(-f_{i, k}^{d}+\left(1-2 f_{i, k}^{d}\right) \delta_{i}\right)
$$

where $w_{n}=1-w_{d}$ and $\delta_{i} \in[0,1]$ is a random number that characterize the influence of inaccuracy and uncertainty. The component $f_{i, k}^{d}$ is related to perception abilities of the ith agent and is defined as:

$$
f_{i, k}^{d}=w_{d}\left(1-\frac{1}{1+e^{-\alpha\left(d_{i, k}^{0}-s_{r, i}\right)}}\right)
$$

Balancing the influence of the random and detection part of the model we can simulate reliability of particular agents.

\subsection{Communication}

Agents are provided with communication devices that allow the exchange of information and coordination of mutual actions. It is also assumed that the 
communication abilities of agents are limited. The limitations for the purpose of this study are narrowed down to the range of the communication devices. That means the given agent is able to communicate only with those agents that are closer than some threshold value. Let us define the set, which determines the those agents that ith agent is able to communicate with:

$$
\Phi_{i}=\{j\}, \quad j \in R \wedge j \neq i
$$

It must be stressed that two agents are in communication only when the transmission of data can be made in both directions. The agent can receive information and its transmission can be received by the other one. This happens only if the following is satisfied:

$$
\mathrm{j} \in \Phi_{i} \Leftrightarrow d_{i, j}^{r}<b_{i} \wedge d_{i, j}^{r}<b_{j}
$$

where $b_{i / j}$ denotes the range of communication devices of the ith and $j$ th agent, while the value $d_{i, j}^{r}$ in (8) is the distance between the ith and jth agent.

\section{$3 \quad$ Agent Reliability Assessment}

As was mentioned before, the information fusion idea is based on the assumption that each agent is able to evaluate (during the task performance) its reliability related to the acquired sensory data correctness. In this section the method of reliability assessment will be presented. Let us first distinguish the discrete moment in the MAS operation and let us denote it by $n=0,1,2, \ldots$.In the beginning of the MAS operation $(n=0)$ the reliability of ith agent is equal to some predefined value:

$$
c_{i}(n=0)=c_{i 0} \in[0,1]
$$

Let us assume that $i t h$ agent in the moment $n$ detects the set of objects defined by (3). We will refer to this set hereafter as:

$$
O_{i}(n)=\{j\}, \quad i \in R, j \in O \wedge O_{i} \subseteq O
$$

After detecting the objects defined by (10), the certainty factor is determined for each object detected according to the function (5), let us denote this estimated value as:

$$
\hat{p}_{i, j}, j \epsilon O_{i}(n)
$$

While performing the task and picking up the objects (10) the agent is able to confront the real state of the process with its predictions. Let us introduce the factor that defines an error of the certainty assessment done by the ith agent with respect to the $j t h$ object detected:

$$
e_{i, j}=\left|\hat{p}_{i, j}-p_{i, j}\right|
$$


where $p_{i, j} \in\{0,1\}$ in (12) denotes a detection correctness of the $j t h$ object. If the object was detected correctly $p_{i, j}$ takes the value 1 and 0 otherwise. Using the value of (12) we can determine the agent reliability based on a single picking up operation. The reliability value is calculated as a function of the (12). In this research an sigmoid function was applied to assign the value of reliability on the basis of the detection correctness error. Hence the reliability factor defined for ith agent using the $j t h$ picking up experiment is defined as:

$$
c_{i, j}=1-\frac{1}{1+e^{-\beta\left(e_{i, j}-e_{i 0}\right)}}
$$

where $\beta$ parameter tunes the steepness of the curve around the threshold $e_{i 0}$. This parameter defines the maximal acceptable error (12), by which it is possible to set the agent reliability at the level of 1 . Applying the equation (13) to each object from the set (10) it is possible to determine the reliability of the agent at the $n t h$ stage of the system operation. In this study it is obtained by averaging results:

$$
c_{i}^{o_{i(n)}}=\frac{1}{k} \sum_{k=1}^{K} c_{i, k}, K=\overline{\bar{O}}_{l}(n)
$$

The detection and recognition process is performed on the basis of the data collected in the single operation cycle of the sensory system. Therefore calculating the agent reliability as the average given by (14) seems to be valid approach. Finally, the reliability of the ith agent at the $n t h$ stage of the process can be evaluated as an exponential moving average with a time window of length $Z$. It can be determined recursively using the following formula:

$$
\begin{gathered}
c_{i}(0)=c_{i}^{o_{i}(0)} \\
c_{i}(n)=\lambda c_{i}^{o_{i}(n)}+(1-\lambda) c_{i}(n-1), \quad \text { for } n>0
\end{gathered}
$$

where $\lambda$ is a constant smoothing factor which value can be from 0 to 1 . It represents the weighting factors applied to the values calculated in the past. The higher value of $\lambda$ the faster discounting older values is. In this study this coefficient is expressed in terms of the time window length:

$$
\lambda=\frac{2}{Z+1}
$$

Applying the above reasoning to every agent, we can assess their reliability at each stage of the process.

\section{Data Fusion}

As was stated in the previous sections in the MAS information about the process performed is distributed among multiple agents. Due to different perception 
abilities, location and possible malfunctions of the sensory devices, the information about the same part of the process (in the case of this study - objects) can be different. Since agents are able to communicate each other and exchange data, the information fusion can be made. Various information about the same part of the process can be used to increase the agents certainty. In the case discussed, agents exchange information about the objects detected as well as the certainty of the detection fact. Since they share the common resources, the same object can be detected by multiple agents. So the question arises how make a fusion of information collected by different agents. In this study an application of the reliability factor was applied. It was assumed that each agent is able to assess its reliability by confronting its certainty estimation with the real state of the process. Let us define the set $R_{k}$ of agents, and their certainties related to the fact of detecting the object $k$ :

$$
\hat{P}_{k}=\left\{\hat{p}_{i, k}\right\}, \quad i \in R_{k}
$$

Using the reliability notion, it is possible to make fusion of certainty by weighted averaging the data provided by the agents. Hence the common certainty of agents defined in $R_{k}$, about the object $k t h$ is calculated as:

$$
p_{R_{k}, k}(n)=\frac{1}{\sum_{i \in R_{k}} c_{i, k}} \sum_{i \in R_{k}} c_{i, k}(n) \hat{p}_{i, k}
$$

The reliability of each agent is a function of the weighting coefficient. The higher reliability the greater influence of the information collected by the agent on the final certainty value.

\section{Simulation}

In order to verify and evaluate the quality of the method presented, a simulation experiment was arranged. Two robotic-agents are placed inside an rectangular workspace of size $500 \times 500[\mathrm{~m}]$. Each agent is equipped with a sensory system which is able to detect objects in the area defined by the angle of view and the range of the sensors. The values of the range and angle are set to $(90[\mathrm{~m}], 75[\mathrm{deg}])$ and $(110[\mathrm{~m}], 95[\mathrm{deg}])$ for the first and second agent correspondingly. Inside the workspace there are 100 objects placed on locations selected in a random way. Figure 2 presents the experiment arrangement. The goal of the agents is efficient collecting objects. The agents are able to communicate each other and exchange information about the objects detected. Moreover it is assumed that agents exchange information about their reliability self-assessment. 


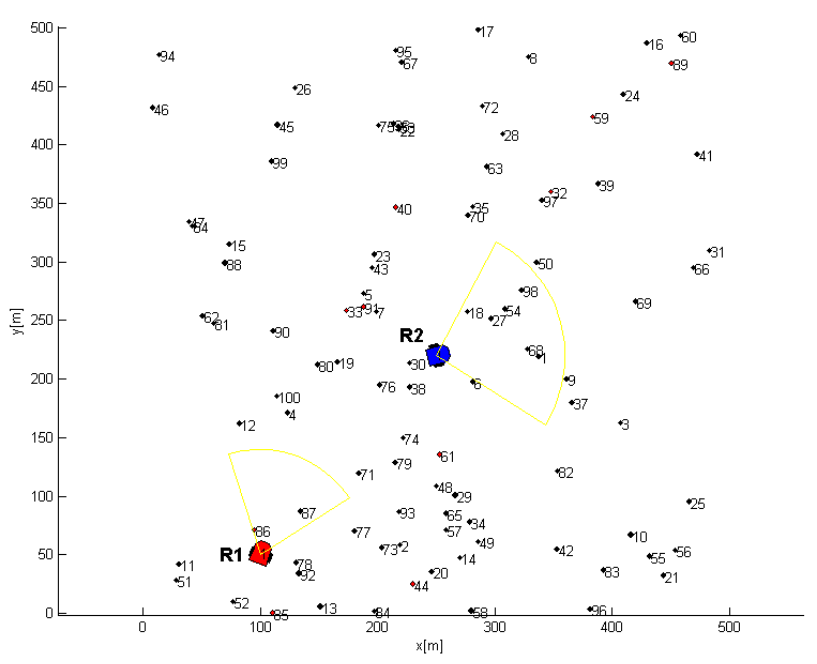

Fig. 2. The simulation experiment arrangement

The aim of the first part of the simulation experiment was to verify the procedure quality of the reliability assessment. The experiment was as follows. The agent was repeating the procedure detect-collect 15 times. The reliability of the agent was modeled according to the (5) by changing the influence of the random component. The simulation was performed for three values of $w_{d}=0.7,0.5,0.05$. The results of the experiment are presented in the fig.3.

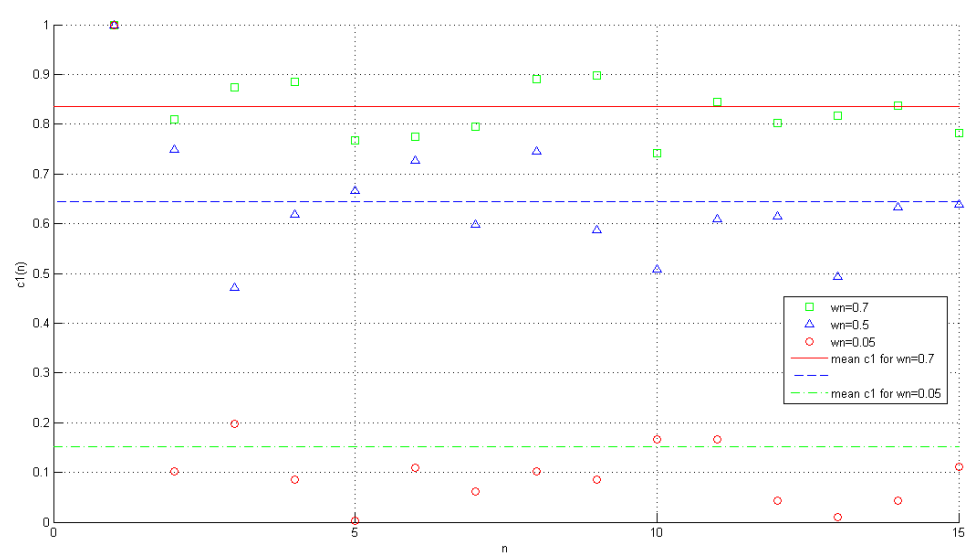

Fig. 3. The agent reliability assessment 
We can observe that with the decrease of $w_{n}$ (what corresponds to increasing the random component) the reliability assessment decrease what means the evaluation method works correctly.

Next part of experiment was arranged to evaluate an reliability influence on the effectiveness of the pick up collection task performance. The first agent was performing 15 detect-collect cycles. The number of objects collected $N_{c o l}$ was counted. The procedure was repeated 5 times. The average number of objects collected in 5 simulation runs was the measure of the method effectiveness. The procedure described above was done for three different values of parameter $w_{n}$. The results are gathered in the tabular (fig.4).

\begin{tabular}{|c|c|c|}
\hline \multirow{2}{*}{ No. } & \multicolumn{2}{|c|}{$\mathrm{N}=10, \mathrm{RP}=5$} \\
\cline { 2 - 3 } & $\mathrm{w}_{\mathrm{n}, 1}$ & $\mathrm{~N}_{\mathrm{col}}$ \\
\hline 1 & 1 & 42 \\
\hline 2 & 0.5 & 31 \\
\hline 3 & 0 & 9 \\
\hline
\end{tabular}

Fig. 4. Results of the second experiment

As can be seen the quality of the sensing, which is related to the reliability has a direct influence on the effectiveness of the task performed. The last part of simulation research was to asses influence of exchanging information on the agent effectiveness improvement. In this experiment two agents were performing the task simultaneously. The agents were in permanent communication. The first agent was modeled as the one of high reliability. The reliability of the second one was set by the parameter $w_{n}$ to values 0.5 and 0 . We can see that thanks to the communication and information fusion effectiveness of performing the task was improved (compare fig.4 and fig.5).

\begin{tabular}{|l|l|l|l|}
\hline \multirow{2}{*}{ No. } & \multicolumn{3}{|c|}{$\mathrm{N}=10, \mathrm{RP}=5$} \\
\cline { 2 - 4 } & $\mathrm{W}_{\mathrm{n}, 1}$ & $\mathrm{~W}_{\mathrm{n}, 2}$ & $\mathrm{~N}_{\mathrm{col}, 1}$ \\
\hline 1 & 0.5 & 1 & 36 \\
\hline 2 & 0 & 1 & 13 \\
\hline
\end{tabular}

Fig. 5. Results of the third experiment

That proves that proper data fusion in MAS can improve the quality of the system operation. 


\section{Conclusion}

This paper addressed the problem of information fusion in MAS. The method of combining data provided by intercommunicated agents is discussed. The approach presented is based on reliability of particular agents. The level of reliability is evaluated by each agent while performing the task by confronting its data collected with the real state of the process. This approach is very simple to implement what is its great advantage. The functioning of the data fusion algorithm is illustrated using an example of pick up and collection task. Nevertheless the method is flexible and can be applied to any MAS in which agents are able to assess its reliability using different sensory systems, the vision systems for instance. Some relevant simulation examples are presented to prove the effectiveness of the approach presented.

\section{References}

[1] Cheng, X., Shen, J., Liu, H., Gu, G.: Multi-robot Cooperation Based on Hierarchical Reinforcement Learning. In: Shi, Y., van Albada, G.D., Dongarra, J., Sloot, P.M.A. (eds.) ICCS 2007, Part III. LNCS, vol. 4489, pp. 90-97. Springer, Heidelberg (2007)

[2] Harmati, I., Skrzypczyk, K.: Robot team coordination for target tracking using fuzzy logic controller in game theoretic framework. Robotics and Autonomous Systems 57(1) (2009)

[3] Jones, C., Mataric, M.: Adaptive Division of Labor in Large-Scale Minimalist Multi-Robot Systems. In: Proc. of IEEE/RSJ International Conference on Intelligent Robots and Systems, Las Vegas, pp. 1969-1974 (2003)

[4] Kaminka, G.A., Erusalimchik, D., Kraus, S.: Adaptive Multi-Robot Coordination: A Game-Theoretic Perspective. In: Proc. of IEEE International Conference on Robotics and Automation, Anchorage Convention District, Anchorage, Alaska, USA (2002)

[5] Kok, J.R., Spaan, M.T.J., Vlassis, N.: Non-communicative multi-robot coordination in dynamic environments. Robotics and Autonomous Systems 50(2-3), 99-114 (2005)

[6] Klusch, M., Gerber, A.: Dynamic coalition formation among rational agents. IEEE Intelligent Systems 17(3), 42-47 (2002)

[7] Kraus, S., Winkfeld, J., Zlotkin, G.: Multiagent negotiation under time constraints. Artificial Intelligence 75, 297-345 (1995)

[8] Kraus, S.: Negotiation and cooperation in multiagent environments. Artificial Intelligence 94(1-2), 79-98 (1997)

[9] Mataric, M., Sukhatme, G., Ostergaard, E.: Multi-Robot Task Allocation in Uncertain Environments. Autonomous Robots 14, 255-263 (2003)

[10] Schneider-Fontan, M., Mataric, M.J.: Territorial Multi-Robot Task Division. IEEE Transactionson Robotics and Automation 14(5), 815-822 (1998)

[11] Winkfeld, K.J., Zlotkin, G.: Multiagent negotiation under time constraints. Artificial Intelligence (75), 297-345 (1995) 
[12] Wooldridge, M.: An Introduction to Multiagent Systems. Johnn Wiley and Sons Ltd., UK (2009) ISBN:978-0-470-51946-2

[13] Vail, D., Veloso, M.: Dynamic Multi-Robot Coordination. In: Schultz, A., et al. (eds.) Multi Robot Systems: From Swarms to Intelligent Automata, vol. II, pp. 8798. Kluwer Academic Publishers, The Netherlands (2003)

[14] Cheng, X., Shen, J., Liu, H., Gu, G.: Multi-robot Cooperation Based on Hierarchical Reinforcement Learning. In: Shi, Y., van Albada, G.D., Dongarra, J., Sloot, P.M.A. (eds.) ICCS 2007, Part III. LNCS, vol. 4489, pp. 90-97. Springer, Heidelberg (2007)

[15] Gałuszka, A., Pacholczyk, M., Bereska, D., Skrzypczyk, K.: Planning as Artificial Intelligence Problem-short introduction and overview. In: Nawrat, A., Simek, K., Świerniak, A. (eds.) Advanced Technologies for Intelligent Systems of National Border Security. SCI, vol. 440, pp. 95-104. Springer, Heidelberg (2013)

[16] Jędrasiak, K., Bereska, D., Nawrat, A.: The Prototype of Gyro-Stabilized UAV Gimbal for Day-Night Surveillance. In: Nawrat, A., Simek, K., Świerniak, A. (eds.) Advanced Technologies for Intelligent Systems of National Border Security. SCI, vol. 440, pp. 107-116. Springer, Heidelberg (2013)

[17] Galuszka, A., Bereska, D., Simek, K., Skrzypczyk, K., Daniec, K.: Application of graphs theory methods to criminal analysis system. Przeglad Elektrotechniczny $86(9), 278-283$ (2010) 